\title{
Histological, Immunohistochemical, Morphometric and Molecular Changes in the Wall of Human Varicose Veins
}

\author{
DOAA MAHMOUD SHUAIB, M.D. \\ The Department of Anatomy\& Embryology, Faculty of Medicine, Cairo University
}

\begin{abstract}
Background: Varicose veins (VVs) are a major chronic venous disease, yet the underlying mechanisms are unclear. This disorder is characterized by progressive architectural remodeling of the vessel wall. Recent studies have focused on the role of matrix metalloproteinases (MMPs) in the pathogenesis of VVs.
\end{abstract}

Aim of Study: The present study was assigned to demonstrate the morphologic and morphometric changes in the wall of VVs and to correlate these changes with the expression of MMP-1 and MMP-9.

Material and Methods: A total number of 18 human long saphenous vein samples ( 8 normal and $10 \mathrm{VVs}$ ) were included in the study. The specimens were processed and examined histologically using hematoxylin and eosin, Masson's trichrome and orcein, together with immunohistochemistry for alpha smooth muscle actin (Éø-SMA). The mRNA expression of MMP-1 and MMP-9 was analyzed using real-time polymerase chain reaction (PCR). Quantitative morphometric measurements were carried out by the image analyzer.

Results: The wall of VVs showed damaged endothelial surface, interrupted internal elastic lamina and numerous vasa vasora. There was significant increase in intima and media thickness compared to normal veins. Significant increase in the mean collagen area percent and decrease in elastin area percent was also observed. The mean area percent of É $\varnothing$-SMA immunostaining and count of smooth muscle cells (SMCs) showed significant increase, indicating SMCs hypertrophy and hyperplasia, while the optical density of É $\varnothing-S M A$ immunostaining decreased. These changes were associated with significant increase in mRNA expression of MMP-1 and MMP-9.

Conclusion: Varicose veins showed marked disorganization in all components of the vessel wall (collagen, elastin and SMCs). The associated increase in mRNA expression of MMPs might be one of the molecular mechanisms underlying vascular remodeling.

Key Words: Varicose veins - Elastin-Collagen - Smooth muscle cells - Matrix metalloproteinases.

Correspondence to: Dr. Doaa Mahmoud Shuaib, E-Mail: doaa.shuaib@kasralainy.edu.eg

\section{Introduction}

VARICOSE veins (VVs) are a common health disorder characterized by extensive dilation and tortuosity of the superficial lower limb veins, associated with incompetent valves and venous reflux [1]. If they are untreated, serious complications may occur including venous leg ulcers, thrombophlebitis and deep venous thrombosis [2].

Varicose veins are an important cause of morbidity in young and elderly population [3], with prevalence up to $56 \%$ of men and up to $73 \%$ of women [4]. The most important risk factors include age, family history, hormones, obesity and pregnancy [5]. Despite the high prevalence of VVs, the exact etiology and pathophysiologic mechanisms are unclear $[3,6,7]$.

Normally, the elasticity and distensibility of veins are provided by balance between collagen and elastin contents of the extracellular matrix (ECM) [8]. Moreover, the smooth muscle cells (SMCs) play an important role in maintaining venous wall tone and blood pressure [9]. It was reported that progressive architectural remodeling of the venous wall with alterations in the ECM components and SMCs may contribute to the pathophysiology of VVs $[6,10]$

Matrix metalloproteinases (MMPs) are a group of enzymes, which are secreted by fibroblasts and SMCs and play a crucial role in remodeling of the ECM proteins including collagen and elastin [11] Moreover, they may affect endothelial cells, SMCs migration, proliferation and contraction. They are regulated at the mRNA expression and enzymatic activity levels [2]. Matrix metalloproteinases have been associated with vascular diseases including VVs [1,7]. From several MMPs, it was reported 
that MMP-1 and MMP-9 have a key role in the pathogenesis of VVs [3].

In the reviewed literature, the studies concerning histopathological changes of VVs focused on either ECM or SMCs. Moreover, few studies correlated histological changes in VVs with alterations in the expression of MMPs. The present study aimed to provide detailed morphologic and morphometric changes in the wall of VVs considering ECM components (collagen and elastin) as well as SMCs, and to correlate these changes with mRNA expression of MMP-1 and MMP-9.

\section{Material and Methods}

\section{Materials:}

A total number of 18 human long saphenous vein (LSV) samples were collected from patients admitted to the Vascular Surgery Department, Faculty of Medicine, Cairo University from December 2017 to September 2018. Informed consent was taken from all patients.

The normal non-varicose veins (control) were obtained from residual vein tissues of 8 patients (5 males and 3 females, mean age: $56.75 \pm 4.5$ years, range: 51-64 years), who were undergoing coronary artery bypass grafting surgery. The patients presented no pathology of the venous system and normal veins were previously investigated using colored duplex ultrasound.

Varicose vein samples were obtained from 10 patients (4 males and 6 females, mean age: $51.2 \pm 5.9$ years, range: $42-60$ years), who subjected to LSV stripping with multiple avulsions. Full history taking, preoperative physical examination and duplex ultrasonography were done to confirm the diagnosis of primary VVs before surgery. Patients with history of thrombophlebitis, phlebitis without thrombus, venous obstructions from neoplasms, severe lower limb edema, arterial disease and diabetes were excluded from the study.

\section{Methods:}

Specimen collection: The venous samples (1$2 \mathrm{~cm}$ long) were obtained from the trunk of the LSV below the knee. The specimens were fixed in $10 \%$ neutral buffered formalin, embedded in paraffin wax and cut into 5 mick sections for histological and morphometric study. In addition, the fresh specimens were collected and used for PCR.

\section{A- Histological study:}

The following stains were used:

1- Hematoxylin and eosin (H \& E) stain: For routine histological examination [12].

2- Masson's trichrome stain: For detection of collagen fibers [12].

3- Orcein stain: For demonstration of elastic fibers [12].

4- Alpha smooth muscle actin ( $\alpha$-SMA) immunostaining: For examination of SMCs. Anti-alpha smooth muscle actin monoclocal antibody [EPR5368] was the primary antibody used at a dilution of 11000/ on paraffin sections (Abcam pharmaceuticals). Paraffin sections were dewaxed and subjected to heat mediated antigen retrieval in sodium citrate buffer (10mM Sodium Citrate, $0.05 \%$ Tween 20, pH 6.0). Endogenous peroxidase activity was blocked with $0.3 \%$ $\mathrm{H} 2 \mathrm{O} 2$ in methanol for 20 minutes. Sections were incubated with fetal calf serum for 30 minutes at $37^{\circ} \mathrm{C}$, followed by incubation with the primary antibody overnight at $4^{\circ} \mathrm{C}$. Then they were rinsed in Phosphate Buffer Saline (PBS). The secondary antibody (Horse radish peroxidase labelled biotin antibody) was applied to the slides at $37^{\circ} \mathrm{C}$ for 30 minutes. The sections were then treated with diaminobenzidine- $\mathrm{H} 2 \mathrm{O} 2$ mixture and counterstained with haematoxylin. For negative control, the same steps were followed but with omission of the primary antibody [13].

\section{B- Morphometric study:}

1- The intima and media thickness in H\&E stained sections.

2- The area percent of collagen fibers in Masson's trichrome stained sections.

3- The area percent of elastic fibers in orcein stained sections.

4- The area percent and optical density of $\alpha$-SMA immunostained SMCs.

5- The SMCs count: the number of $\alpha$-SMA immunostained SMCs.

The pervious measurements were done using "Leica Qwin 500C" image analyzer computer system (Cambridge, UK). Ten non overlapping low power fields (x100) were randomly chosen from five sections from each sample. 


\section{C- Measurement of MMP-1 and MMP-9 mRNA expression by quantitative real-time PCR:}

Pieces from the venous samples were homogenized and total RNA was extracted using SV Total RNA Isolation System (Promega, Madison, WI, USA) according to the instructions of the manufacturer. The purity and concentration of RNA were measured with an ultraviolet spectrophotometer. Complementary cDNA was synthesized from RNA using SuperScript ${ }^{\circledR}$ III First-Strand Synthesis System as described in the protocol of the manufacturer (\#K1621, Fermentas, Waltham, MA, USA). Amplification and analysis of real-time PCR were done using an Applied Biosystem instrument with software version 3.1 (StepOne TM,USA). Primers targeting MMP-1 and MMP-9 genes were selected from the literature $[\mathbf{1 5 , 1 6 ]}$. All values were normalized to beta actin and reported as fold change (Table 1).

Table (1): The primer sequences for the studied genes.

\begin{tabular}{lll}
\hline Isoform & Forward primer & Reversed primer \\
\hline MMP-1 & 5'-ACCTTCAGTGG & 5'-ATCCGTGTAGCA \\
& TGATGTTCAGC-3' [15] & CATTCTGTCC-3' [15] \\
\multirow{2}{*}{ MMP-9 } & 5'-GGTGGACCG & 5'-GCCCACCT \\
& GATGTTCCC -3' [16] & CCACTCCTCC -3' [16] \\
\multirow{3}{*}{-actin } & 5'-ACACTGTGCCC & 5'-GGAGGGGCCGG \\
& ATCTATGAGG -3' [16] & ACTCGTCATACT -3' [16] \\
\hline
\end{tabular}

MMP-1 : Matrix metalloproteinase-1.

MMP-9 : Matrix metalloproteinase-9.

\section{Statistical analysis:}

Data was entered on the computer using "Microsoft Office Excel Software" program (2010) for windows. Data was then transferred to the Statistical Package of Social Science Software program, version 23.0 (IBM SPSS Statistics for Windows, Version 23.0. Armonk, NY: IBM Corp.) to be statistically analyzed. Data presented as mean \pm standard deviation (SD). Comparison between the two groups was done using student $\mathrm{t}$ (for normal data) or Mann Whitney tests (for skewed data). $p$-values less than 0.05 were considered statistically significant.

\section{Results}

\section{A- Histological results:}

\section{Hematoxylin \& eosin stained sections:}

Histological examination of $\mathrm{H} \& \mathrm{E}$ stained sections of control group showed the normal his- tology of the LSV, which was composed of three tunicae (from the lumen to the periphery: The intima, the media and the adventitia). Tunica intima was formed of a thin layer of SMCs covered by endothelium and separated from the underlying media by well-developed internal elastic lamina (IEL). The media consisted of circular-oriented SMCs arranged in concentric lamellae and embedded in ECM. The adventitia was composed of connective tissue containing vasa vasora (Figs. 1a, b,c).

In VVs, marked variability was observed in the organization of the vessel wall. There was apparent increase in the thickness of the intima, which appeared folded with an irregular surface. In some areas, deep clefts were observed due to the highly folded intima. Discontinuity of endothelial surface with damaged endothelium was also encountered. The media was apparently thick and showed hypertrophied SMCs, which lost their regular arrangement. The adventitia exhibited an increase in the number of vasa vasora, which were surrounded by irregular collagen fibers (Figs. 1d,ej).

\section{Masson's trichrome stained sections:}

In normal LSV, moderate amount of collagen fibers were observed in the intima and between the SMCs of the media. Continuous IEL was detected (Figs. 2a,b). Varicose vein sections stained with Masson's trichrome showed large amount of collagen fibers separating the hypertrophied SMCs in the media. The intima showed longitudinally arranged SMCs separated by collagen fibers and the IEL was fragmented (Figs. 2c,d).

\section{Orcein stained sections:}

The normal LSV showed moderate amount of elastic fibers in the media and the adventitia. Welldeveloped IEL was observed (Figs. 3a,b,c). In VVs, the media showed fewer elastic fibers, which were short and scattered. The adventitia exhibited disorganization in the pattern of elastic fibers, which appeared clumped. The IEL was interrupted (Figs. 3d,ej).

\section{Alpha smooth muscle actin immunostained sections:}

In normal LSV, there was strong $\alpha$-SMA immunoreaction in the SMCs of the media (Figs. $4 a, b)$. In VVs, the SMCs of the media were hypertrophied and showed weak $\alpha$-SMA immunoexpression (Figs. 4c,d). 

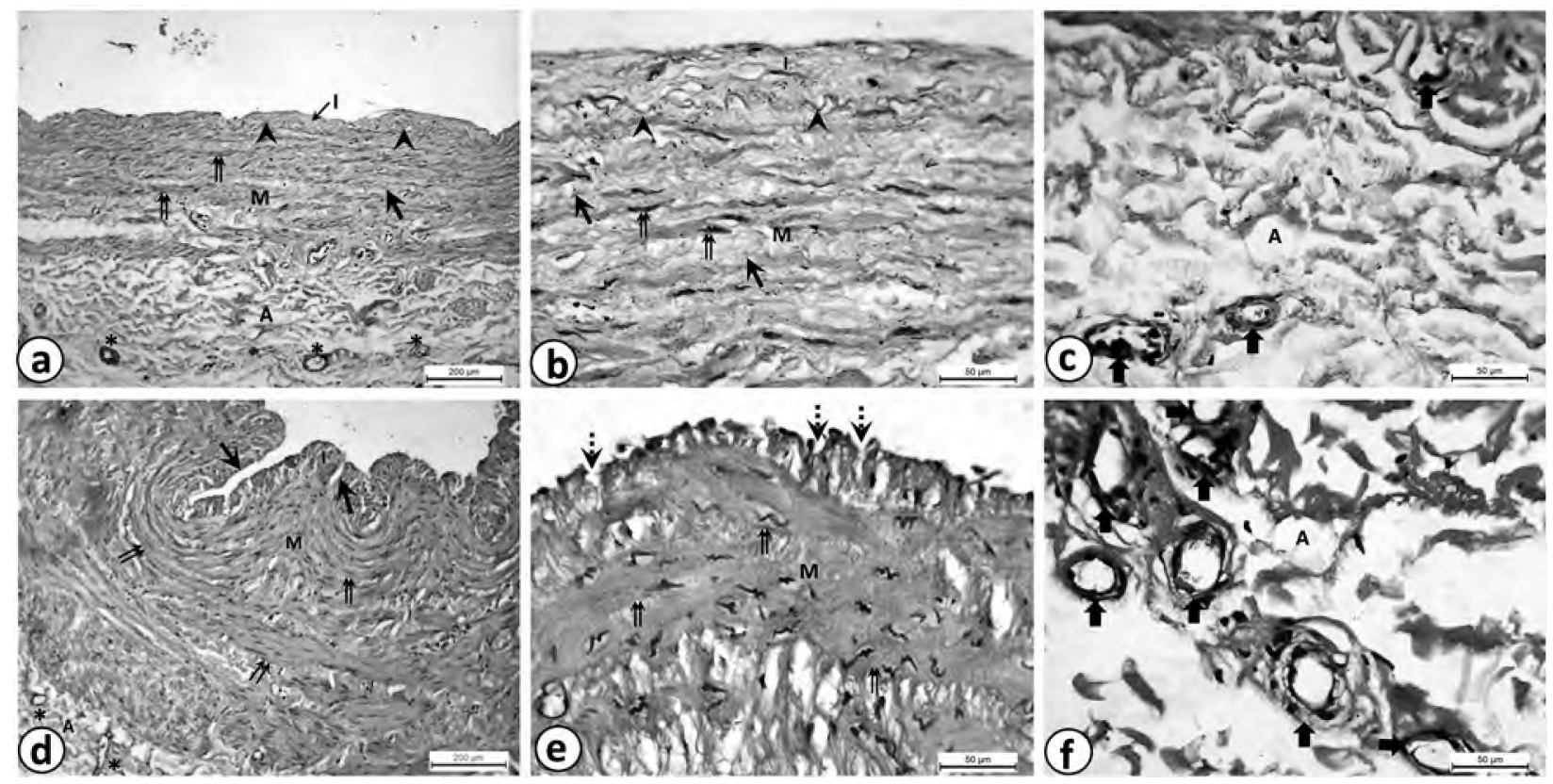

Fig. (1): Photomicrographs of transverse sections of control (a,b,c) and varicose (d,e,f) LSVs:

(a): Control LSV shows tunica intima (I), media (M) and adventitia (A). Well-developed IEL (arrow heads), SMCs (double arrows) and elastic fibers (arrow) are illustrated. Vasa vasora (asteriks) are shown in the adventitia (H\&E X100).

(b): Higher magnification shows tunica intima (I), media (M), IEL (arrow heads), SMCs (double arrows) and elastic fibers (arrows) (H\&E $\mathrm{X} 400$ ).

(c): Higher magnification shows vasa vasora (thick arrows) in the tunica adnititia (A) (H\&E X400).

(d): Varicose LSV shows deep clefts (arrows) in the thickned intima (I), hypertophied SMCs (double arrows) in the media (M) and multiple vasa vasora (asteriks) in the advintitia (A) (H\&E X100).

(e): Higher magnification shows interrupted endothelial surface (dotted arrows). Hypertophied SMCs (double arrows) are demonstrated in the media (M) (H\&E X400).

(f): Higher magnification shows increased number of vasa vasora (thick arrows) in the adventitia (A) (H\&E X400).
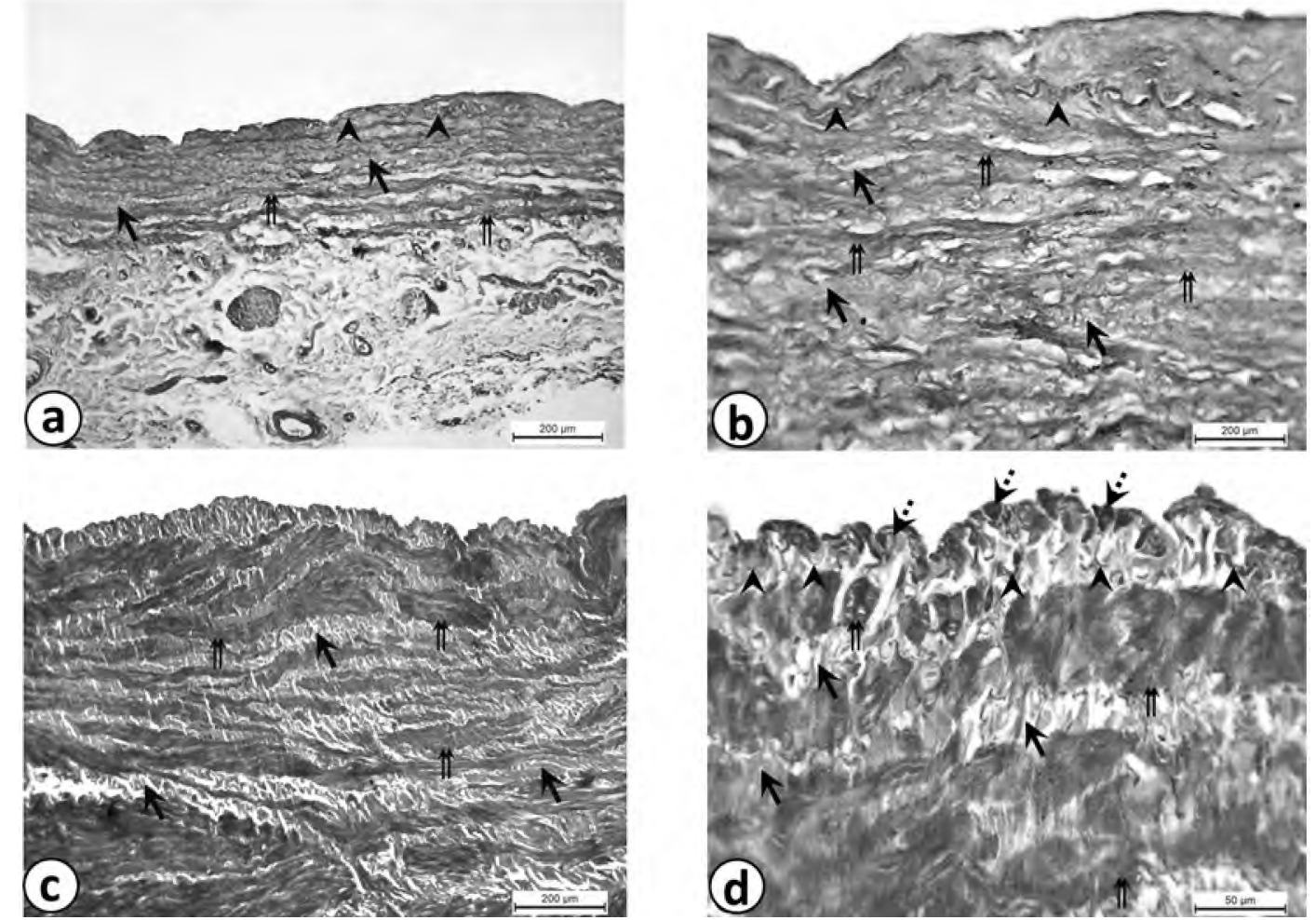

Fig. (2): Photomicrographs of transverse sections of control $(a, b)$ and varicose (c,d) LSVs:

(a): Control LSV shows moderate amount of collagen bundles (arrows) between SMCs (double arrows). Continuous IEL (arrow heads) is demonstrated (Masson's Trichrome X100).

(b): Higher magnification shows moderate amount of collagen bundles (arrows) between SMCs (double arrows). Intact IEL (arrow heads) is shown (Masson's Trichrome X400).

(c): Varicose LSV shows hypetropied SMCs (double arrows) separated by large amount of collagen fibers (arrows) (Masson's Trichrome X100).

(d): Higher magnification shows multiple longitudinal SMCs (dotted arrows) in the intima and interrupted IEL (arrow heads). The hypetropied SMCs (double arrows) in the media are separated by thick collagen fibers (arrows) (Masson's Trichrome X400). 

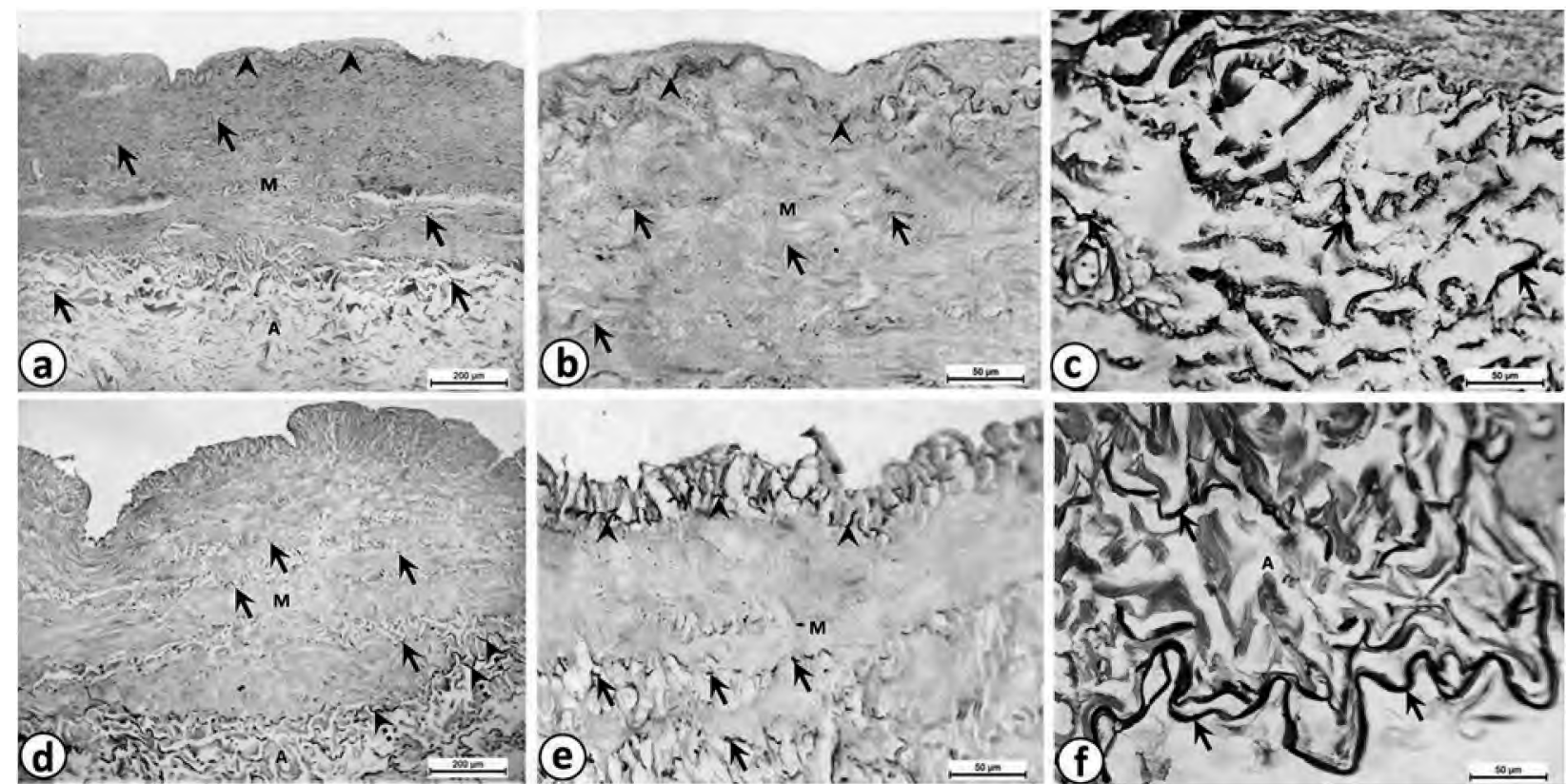

Fig. (3): Photomicrographs of transverse sections of control $(a, b, c)$ and varicose (d,e,f) LSVs:

(a): Control LSV shows moderate amount of elastic fibers (arrows) in the media (M) and advintitia (A). Well-developed continous IEL (arrow heads) is demonstrated (Orcein X100).

(b): Higher magnification shows intact IEL (arrow heads) and moderate amount of elastic fibers (arrows) in the media (M) (Orcein X400).

(c): Higher magnification shows moderate amount of elastic fibers (arrows) in the advinitia (A) (Orcein X400).

(d): Varicose LSV shows short and fragmented elastic fibers (arrows) in the media (M). Clumped elastic fibers (interrupted arrows) are illustrated in the adventitia (A) (Orcein X100).

(e): Higher magnification shows interrupted IEL (arrow heads). Scattered elastic fibers (arrows) are shown in the media (M) (Orcein X400).

(f): Higher magnification shows clumped elastic fibers (arrows) in the advinitia (A) (Orcein X400).
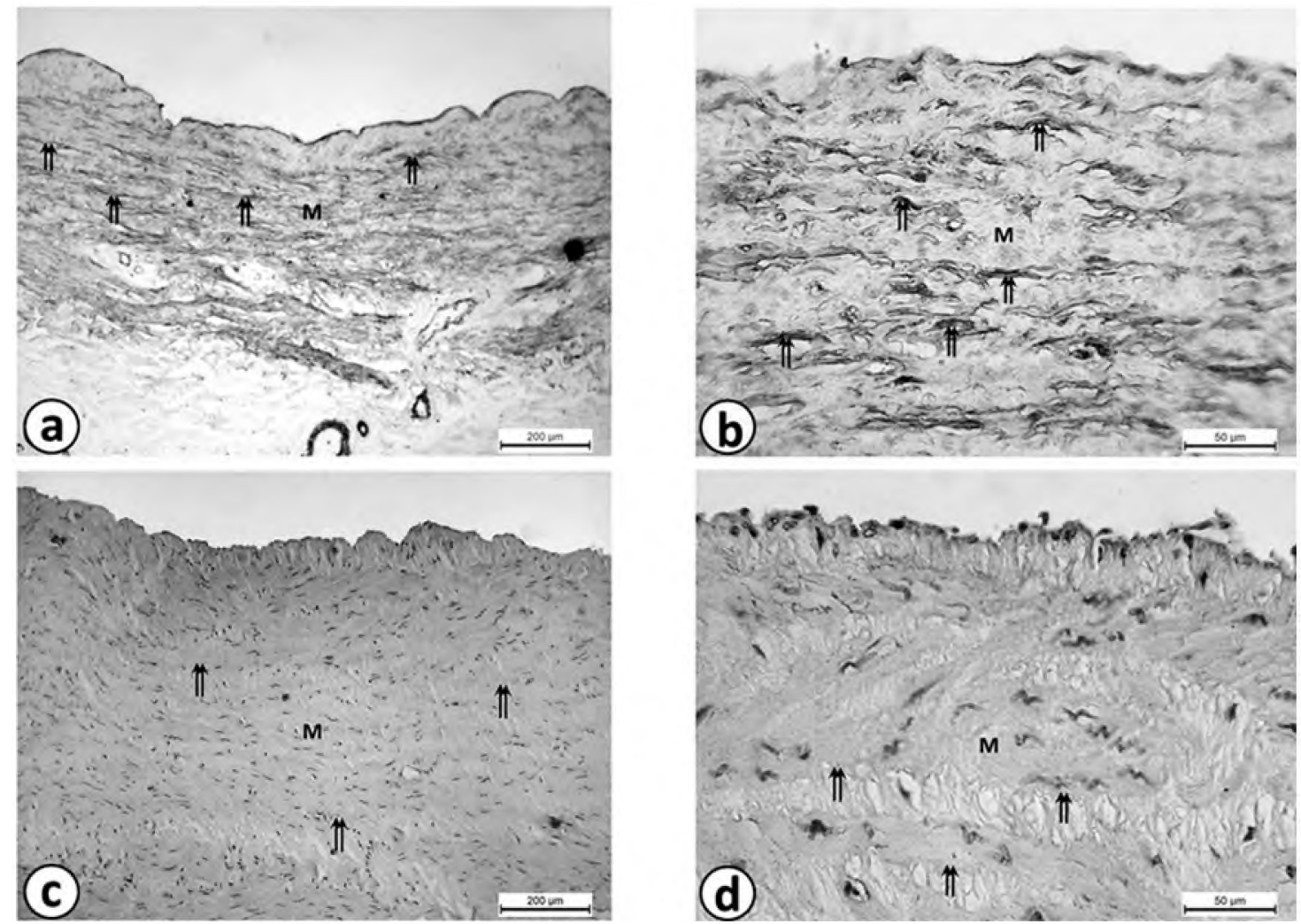

Fig. (4): Photomicrographs of transverse sections of control $(a, b)$ and varicose (c,d) LSVs:

(a): Control LSV shows strong $\alpha$-SMA immunoreaction in the SMCs (double arrows) of the media (M) ( $\alpha$-SMA X100).

(b): Higher magnification of the media (M) shows strong $\alpha$-SMA immunoexpression in the SMCs (double arrows) ( $\alpha$-SMA X400).

(c): Varicose LSV shows hypertophied SMCs with faint Éø-SMA immunoexpression (double arrows) in the media (M) ( $\alpha-$ SMA X100).

(d): Higher magnification of the media (M) shows faintly immunostained hypertrophied SMCs (double arrows) ( $\alpha$-SMA X400). 


\section{B- Morphometric results:}

\section{1- The intima, and media thickness:}

There was significant increase in the mean thickness of intima and media in VVs compared to normal LSVs (Table 2).

\section{2-The area percent of collagen and elastic fibers:}

There was statistical significant increase in the mean area percent of collagen fibers in the wall of VVs compared to normal veins. The mean area percent of elastic fibers showed statistical significant decrease in the wall of VVs compared to normal veins (Table $3)$.

\section{3- The area percent, optical density and count of $\alpha$ - SMA immunostained SMCs:}

Varicose veins showed significant increase in the mean area percent of $\alpha$-SMA immunostained SMCs compared to normal veins. The mean optical density of $\alpha$-SMA immunoreactivity in VVs showed significant decrease compared to normal veins. There was significant increase in the mean count of SMCs in VVs compared to non-varicose veins (Table 4).

\section{$C$ - Determination of MMP-1 and MMP-9 $m R N A$}

expression in the venous wall:

There was significant increase in mRNA expression of MMP-1 and MMP-9 in the tissue of VVs compared to normal veins (Table 5).

Table (2): The mean thickness of intima and media in normal and VVs.

\begin{tabular}{lccc}
\hline & $\begin{array}{c}\text { Normal veins } \\
\text { Mean } \pm \text { SD }\end{array}$ & $\begin{array}{c}\text { Varicose veins } \\
\text { Mean } \pm \text { SD }\end{array}$ & $p$-value \\
\hline Intima thickness $(\mu \mathrm{m})$ & $29.523 \pm 3.551$ & $54.142 \pm 5.732$ & $0.000^{*}$ \\
Media thickness $(\mu \mathrm{m})$ & $257.535 \pm 43.86$ & $580.537 \pm 59.019$ & $0.000^{*}$ \\
\hline
\end{tabular}

${ }^{*} p$-value significant (Mann Whitney test).

Table (3): The mean area percent of collagen and elastic fibers in normal and VVs.

\begin{tabular}{lccc}
\hline & $\begin{array}{c}\text { Normal veins } \\
\text { Mean } \pm \text { SD }\end{array}$ & $\begin{array}{c}\text { Varicose veins } \\
\text { Mean } \pm \text { SD }\end{array}$ & $p$-value \\
\hline Collagen area \% & $21.234 \pm 1.912$ & $40.896 \pm 1.904$ & $0.009 *$ \\
Elastin area \% & $19.416 \pm 2.598$ & $11.018 \pm 1.336$ & $0.000 \#$ \\
\hline
\end{tabular}

${ }^{*} p$-value significant (Mann Whitney test).

$\# p$-value significant (Student $t$-test)

$\%$ : Percent.
Table (4): The mean area percent, optical density and count of $\alpha$-SMA immunostained SMCs in normal and VVs.

\begin{tabular}{llll}
\hline & $\begin{array}{c}\text { Normal veins } \\
\text { Mean } \pm \text { SD }\end{array}$ & $\begin{array}{c}\text { Varicose veins } \\
\text { Mean } \pm \text { SD }\end{array}$ & $p$-value \\
\hline$\alpha$-SMA area $\%$ & $38.622 \pm 2.933$ & $58.246 \pm 2.344$ & $0.000 \#$ \\
$\alpha$-SMA optical density & $0.508 \pm 0.015$ & $0.254 \pm 0.015$ & $0.009 *$ \\
SMCs count & $176 \pm 16.078$ & $472 \pm 21.966$ & $0.009 *$ \\
\hline
\end{tabular}

*p $p$-value significant (Mann Whitney test).

$\# p$-value significant (Student $t$-test)

$\%$ : Percent; $\alpha$-SMA: Alpha smooth muscl eactin.

SMCs: smooth muscle cells.

Table (5): The mean mRNA expression of MMP-1 and MMP9 in normal and VVs.

\begin{tabular}{llll}
\hline & $\begin{array}{c}\text { Normal veins } \\
\text { Mean } \pm \text { SD }\end{array}$ & $\begin{array}{l}\text { Varicose veins } \\
\text { Mean } \pm \text { SD }\end{array}$ & $p$-value \\
\hline MMP-1 mRNA expression & $1.03 \pm 0.019$ & $2.183 \pm 0.3$ & $0.002^{*}$ \\
MMP-9 mRNA expression & $1.003 \pm 0.004$ & $2.933 \pm 0.394$ & $0.002^{*}$ \\
\hline
\end{tabular}

${ }^{*} p$-value significant (Mann Whitney test).

MMP-1: Matrix metalloproteinase-1.

MMP-9: Matrix metalloproteinase-9.

\section{Discussion}

Varicose veins are a common health problem characterized by extensive venous dilation and remodeling, yet the underlying mechanism is not fully understood [7]. The present study was assigned to demonstrate the histological and immunohistochemical changes in the wall of VVs and to correlate these changes with mRNA expression of MMP1 and MMP-9.

In the present study, there was an increase in intima thickness in VVs compared to normal veins. In some areas, the thickened intima was markedly folded forming deep clefts. This finding was consistent with Surendran et al. [10], who reported that "neointima" is formed as a result of migration and rearrangement of SMCs. Desquamated and interrupted endothelium was observed in the wall of VVs in this study, which was in accordance with Surendran et al. [10] and Birdina et al. [17]. It was emphasized that injury of endothelial cells in VVs triggers leukocytic infiltration and inflammation, which leads to further damage of the venous wall [18].

In the current work, the wall of VVs showed extensive remodeling in ECM architecture. The IEL was disrupted and the elastic fibers of the 
media were short and fragmented. This was confirmed by the significant decrease in elastin area percent in the wall of VVs compared to normal veins. In agreement to our findings, fragmentation of elastic fibers and the decrease of total elastin content in VVs have been reported by Xu et al. [14] and Elsharawy et al. [19] respectively. In contrast, Sansilvestri-Morel et al. [20] found that the elastin content may be increased in VVs.

Fragmentation of IEL and disruption of elastic fibers in VVs in the current study might be due to the proteolytic effect of MMP-9 because it is the main enzyme responsible for elastin assembly as reported by Katsuda and Kaji [21]. This result was confirmed by the significant increase in MMP-9 mRNA expression in the tissue of VVs compared to normal veins. Moreover, the clumped elastic fibers in the adventitial layer of VVs in the current work have been also observed by Elsharawy et al. [19]. Those authors attributed this finding to the proteolysis of the vessel wall, which leads to disorganization of elastin pattern. It was emphasized that any change in the amount or regularity of elastin may cause a decrease in the elasticity of the venous wall with subsequent dilation of the vein [22].

Varicose veins in the present work showed disorganized collagen fibers, which were intercalating with the hypertrophied SMCs. Significant increase in the mean collagen area percent was also observed in the wall of VVs compared to non-varicose veins. Similar findings were mentioned by Ducasse et al. [23] and Xu et al. [14] . Contrary, a decrease in collagen in VVs has been observed by Haviarova et al. [24] and no change in collagen content was reported by Venturi et al. [21]. It was reported that disorganization of the ECM in relation to SMCs is more important than total content of elastin and collagen as they inappropriately invade the SMCs layers reducing their contractile function [25].

In the present study, the tunica media showed significant increase in thickness in VVs compared to non-varicose veins due to abnormal proliferation of SMCs and increased deposition of collagen fibers. Hypertrophy of SMCs was confirmed by the significant increase in the mean area percent of positive a-SMA immunostaining in the wall of VVs compared to normal veins. Increased SMCs mass in the wall of VVs has been also reported by Surendran et al. [10], however, Ducasse et al. [23] found a decrease in SMCs. Jacobs et al. [6] suggested that the conflicting variability in collagen, elastin and SMCs contents in VVs might be due to skip lesions and subsequent difficulty in comparing exactly the same section of vein across and within studies.
The mean optical density of a-SMA immunostaining in VVs of the present work was significantly decreased compared to normal veins, indicating a decrease in the content of a-SMA. This finding might be explained by Xu et al. [26], who suggested that the contractile function of the SMCs was impaired in VVs because they were changed from "contractile" to "synthetic or secretory" phenotype. Moreover, it was reported that the SMCs of the secretory phenotype are responsible for synthesis of collagen, which might lead to increased immature ECM and decreased mature ECM, thus affecting the stability and integrity of venous wall [27]

In the present work, there was significant increase in SMCs count in VVs as compared to normal veins. The same finding was observed by $\mathrm{Xu}$ et al. [26], who reported an increase in the proliferation ability of cultured SMCs derived from VVs. In contrast, Wali and Eid [28] suggested that the apparent increase in SMC mass in VVs was due to non-protein content of the muscle. Therefore, they considered it a process of hypertrophy and not hyperplasia.

It was reported that MMPs promote dissolution of adherens junctions between SMCs, which facilitate their proliferation [29]. They also induce the release of growth factors by cleaving the growth factor-binding proteins and matrix molecules [30] Therefore, the significant increase in SMCs count in the present work might be attributed to the stimulatory effect of MMPs on SMCs. This was confirmed by the significant increase in mRNA expression of MMP-1 and MMP-9 in the wall of VVs compared to normal veins.

Regarding SMCs migration, it was mentioned that in VVs, there is overexpression of MMPs as a result of prolonged increase in venous pressure and vein wall tension [18]. The increased expression of MMP-9 is responsible for degradation of elastic fibers and fragmentation of the IEL, which allows migration of SMCs from the media to the intima [19]. Moreover, MMP-1 facilitates cell migration by allowing the SMCs to sense a proteolytic environment and actively move towards the area of degraded matrix [31]. Thus, the significant increase in mRNA expression of MMP-1 and MMP-9 in the wall of VVs found in the current study might explain migration of SMCs and "neointima" formation.

In agreement to our findings, Yan et al. [7] reported significant increase in MMP-9mRNA expression in VVs compared to normal veins. Naik et al. [3] found an increase the expression of MMP- 
1 in all layers of VVs in $92 \%$ of cases using immunohistochemistry. Contrary, Gillespie et al. [15] found an increase in the activity of MMP-1 in VVs, with no change in MMP-1mRNA expression. Gomez et al. [32] reported a decrease in the activity of MMP-1 in VVs. Chen et al. [2] suggested that the variability in the level of MMPs might be due to examination of different segments from different regions of VVs or examining vein samples from patients at different stages of chronic venous disease.

Numerous vasa vasora were observed in the wall of VVs compared to normal veins in the current study. Similar finding has been observed by Birdina et al. [17], who attributed this observation to hypoxia in VVs wall. That the increase in venous pressure and vein wall tension produce compression of the vasa vasora and increased vein wall oxygen requirements. This leads to vein wall hypoxia and increase in the number of vasa vasora to provide oxygen to the hypoxic vein wall [33].

\section{Conclusion:}

Several histological and immunohistochemical changes have been demonstrated in the cellular and ECM components in the wall of VVs. These changes were associated with an increase in the expression of MMPs, which may be one of the molecular mechanisms underlying wall remodeling. Further studies on different venous regions are recommended to declare the role of MMPs in the pathogenesis of VVs.

\section{Compliance with ethical standards:}

Conflict of interest: The author declares no conflict of interest.

\section{References}

1- MACCOLL E. and KHALIL R.A.: Matrix Metalloproteinases as Regulators of Vein Structure and Function: Implications in Chronic Venous Disease. J. Pharmacol Exp Ther., 355 (3): 410-428, doi: 10. 1 124/jpet. 115.227330, 2015.

2- CHEN Y., PENG W., RAFFETTO J.D. and KHALIL R.A.: Matrix Metalloproteinases in Remodeling of Lower Extremity Veins and Chronic Venous Disease. Prog Mol Biol Transl Sci., 147:267-299, doi: 10. 1016/bs. pmbts. 2017.02.003, 2017.

3- NAIK B., KUMAR M., KHANNA A.K. and SUMAN P.K.: Clinico-histopathological study of varicose vein and role of matrix metalloproteinases-1, matrix metalloproteinases- 9 and tissue inhibitor of matrix metalloproteinase1 in varicose vein formation. Indian J Pathol Microbiol., 59 (1): 25-30, doi: 10. 4103/0377-4929.178217, 2016.

4- BEEBE-DIMMER J.L., PFEIFER J.R., ENGLE J.S. and SCHOTTENFELD D.: The epidemiology of chronic venous insufficiency and varicose veins. Ann. Epidemiol., 15: 175-184, 2005.

5- ORTEGA M.A., ASUNSOLOA., 'ALVAREZ-ROCHA M.J., ROMERO B., DE LEON-LUIS J., 'ALVAREZ-MON M., et al.: Remodelling of collagen fibres in the placentas of women with venous insufficiency during pregnancy. Histol Histopathol., 33 (6): 567-576, doi: 10. 14670/HH11-948, 2018

6- JACOBS B.N., ANDRASKA E.A., OBI A.T. and WAKEFIELD T.W.: Pathophysiology of varicose veins. J. Vasc. Surg. Venous. Lymphat. Disord., 5 (3): 460-467, doi: 10. 1016/j.jvsv. 2016.12.01, 2017.

7- YAN L., TANG J., HU X., XU Y., LI K., LIU H., et al.: Imbalance in matrix metalloproteinases and tissue inhibitor of metalloproteinases from splenic veins and great saphenous veins under high hemodynamics. Phlebology, 24: 268355519842432, doi: 10. 1177/0268355519842432, 2019.

8- SOMERS P. and KNAAPEN M.: The histopathology of varicose vein disease. Angiology, 57 (5): 546-555, 2006.

9- TAN J., YANG L., LIU C. and YAN Z.: MicroRNA-26a targets MAPK6 to inhibit smooth muscle cell proliferation and vein graft neointimal hyperplasia. Sci Rep., 7: 46602, DOI: 10. 1038/srep46602, 2017.

10- SURENDRAN S., S. RAMEGOWDA K., SURESH A., BINIL R.A.J., LAKKAPPA R.K., KAMALAPURKAR G., et al.: Arterialization and anomalous vein wall remodeling in varicose veins is associated with upregulated FoxC2-Dll4 pathway. Lab Invest., 96 (4): 399-408, doi: 10.1038/labinvest. 2015. 167, 2016.

11- SERRALHEIRO P., CAIRRAO E., MAIA C.J., JOAO M., ALMEIDA C. and VERDE I.: Effect of TGFbeta1 on MMP/TIMP and TGF-beta1 receptors in great saphenous veins and its significance on chronic venous insufficiency. Phlebology, 32: 334-341, 2017.

12- BANCROFT J.D. and GAMBLE M.: Theory and practice of histological techniques. 6 th edition, Churchill Livingstone, Elsevier, China, 2008.

13- EI-NEFIAWY N.E.: Development of Human Umbilical Vessels in The Second Trimester of Pregnancy: Histological, Immunohistochemical and Morphometric Study. Egyptian Journal of Histology, 40 (2): 226-240, DOI: 10. 21608/ejh. 2017.4124, 2017.

14- XU N., ZHANG Y.Y., LIN Y., BAO B., ZHENG L., SHI G.P., et al.: Increased levels of lysosomal cysteinyl cathepsins in human varicose veins: A histology study. Thromb Haemost., 111 (2): 333-44, doi: 10. 1160/TH13-04-0309, 2014.

15- GILLESPIE D.L., PATEL A., FILATA B., CHANG A., BARNES S., FLAGG A., et al.: Varicose veins possess greater quantities of MMP-1 than normal veins and demonstrate regional variation in MMP-1 and MMP-13. J. Surg. Res., 106:233-238, 2002.

16- JIANG G. and Qi Y.: Detection of MMP-9 and TIMP-3 mRNA expression in the villi of patients undergoing early spontaneous abortion: A report of 30 cases. Exp. Ther. Med., 9 (5): 1939-1943, 2015.

17- BIRDINA J., PILMANE M. and LIGERS A.: The Morphofunctional Changes in the Wall of Varicose Veins. 
Ann. Vasc. Surg., 42: 274-284, doi: 10. 1016/j.avsg. 2016 10. 064, 2017.

18- KUCUKGUVEN A. and KHALIL R.A.: Matrix metalloproteinases as potential targets in the venous dilation associated with varicose veins. Curr. Drug. Targets, 14 (3): 287-324, 2013.

19- ELSHARAWY M.A., NAIM M.M., ABDELMAGUID E.M. and AL-MULHIM A.A.: Role of saphenous vein wall in the pathogenesis of primary varicose veins. Interact Cardiovasc Thorac Surg., 6 (2): 219-224, 2007.

20- SANSILVESTRI-MOREL P., FIORETTI F., RUPIN A., SENNI K., FABIANI J.N., GODEAU G., et al.: Comparison of extracellular matrix in skin and saphenous veins from patients with varicose veins: does the skin reflect venous matrix changes? Clin. Sci. (Lond)., 112 (4): 229239, 2007.

21- KATSUDA S. and KAJI T.: Atherosclerosis and extracellular matrix. J. Atheroscler. Thromb., 10 (5): 267-274, 2003.

22- VENTURI M., BONAVINA L., ANNONI F., COLOMBO L., BUTERA C., PERACCHIA A., et al.: Biochemical assay of collagen and elastin in the normal and varicose vein wall. J. Surg. Res., 60 (1): 245-248, 1996.

23- DUCASSE E., GIANNAKAKIS K., SPEZIALE F., MIDY D., SBARIGIA E., BASTE J.C., et al.: Association of primary varicose veins with dysregulated vein wall apoptosis. Eur. J. Vasc. Endovasc. Surg., 35 (2): 224-229, 2008.

24- HAVIAROVA Z., WEISMANN P., STVRTINOVA V. and BENUSKA J.: The determination of the collagen and elastin amount in the human varicose vein by the computer morphometric method. Gen. Physiol. Biophys., 1: 30-33, 1999.

25- RAFFETTO J.D., QIAO X., BEAUREGARD K.G.,
TANBE A.F., KUMAR A., MAM V., et al.: Functional adaptation of venous smooth muscle response to vasoconstriction in proximal, distal and varix segments of varicose veins. J. Vasc. Surg., 51: 962-971, 2010.

26- XU Y., BEI Y., LI Y. and CHU H.: Phenotypic and functional transformation in smooth muscle cells derived from varicose veins. J. Vasc. Surg. Venous Lymphat Disord., 5 (5): 723-733, doi: 10.1016/j.jvsv. 2017.04.009, 2017.

27- CHEN S., QIN S., WANG M. and ZHANG S.: Expression and significance of NELIN and SM22 $a$ in varicose vein tissue. Exp. Ther. Med., 9:845-849, 2015.

28- WALI M.A. and EID R.A.: Smooth muscle changes in varicose veins: an ultrastructural study. J. Smooth Muscle Res., 37 (5-6): 123-135, 2001.

29- NELSON W.J. and NUSSE R.: Convergence of Wnt, betacatenin, and cadherin pathways. Science, 303: 1483-1487, 2004.

30- ZHANG H., CHALOTHON D., JACKSON L.F., LEE D.C. and FABER J.E.: Transactivation of epidermal growth factor receptor mediates catecholamine-induced growth of vascular smooth muscle. Circ. Res., 95: 989-997, 2004.

31- WANG X. and KHALIL R.A.: Matrix Metalloproteinases, Vascular Remodeling, and Vascular Disease. Adv Pharmacol., 81: 241-330. doi:10.1016/bs.apha.2017.08.002, 2018.

32- GOMEZ I., BENYAHIA C., LOUEDEC L., LESECHE G., JACOB M.P., LONHROIS D. and NOREL X.: Decreased PGE (2) content reduces MMP-1 activity and consequently increases collagen density in human varicose vein. PLoS One, 9 (2):e88021, 2014.

33- ATTA H.M.: Varicose Veins: Role of Mechanotransduction of Venous Hypertension. International Journal of Vascular Medicine., Article ID 538627, doi. org /10. 1155 / 2012 1538627, 2012. 


\section{التغيرات الهستولوجية، الهستوكيميائية الهناعية،

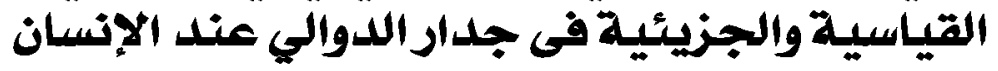

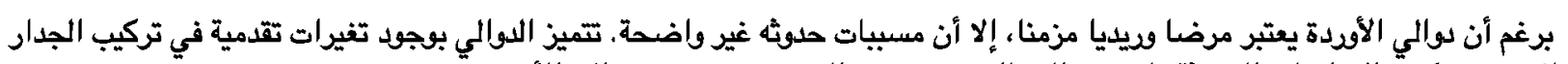

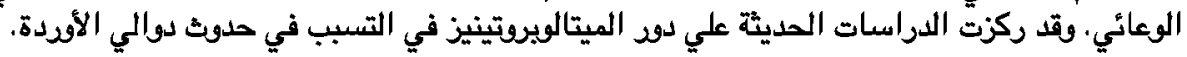

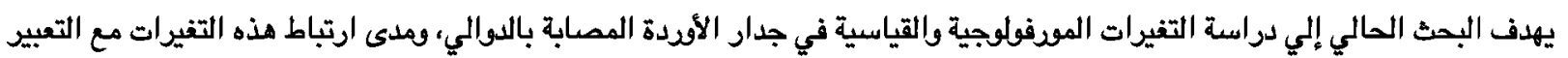

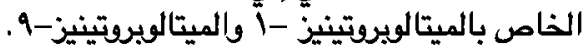

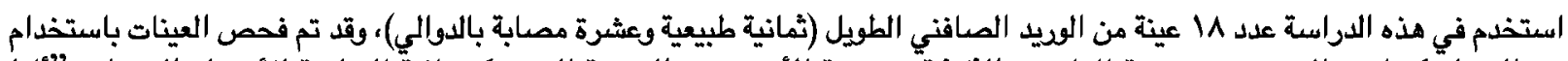

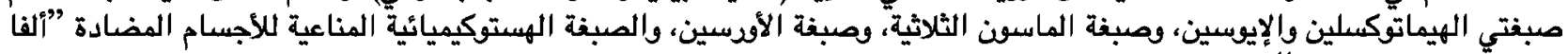

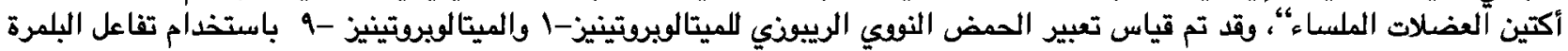

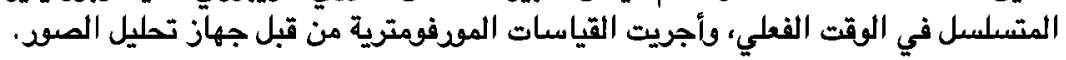

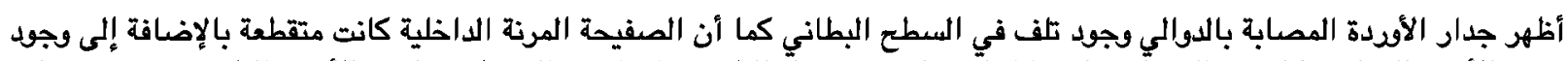

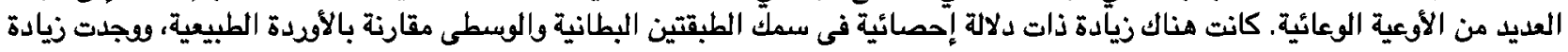

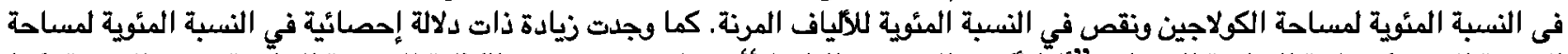

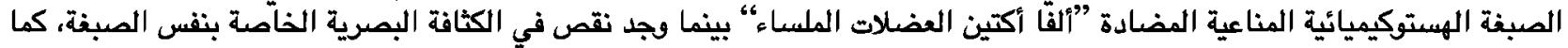

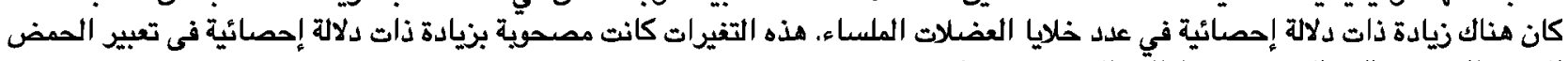

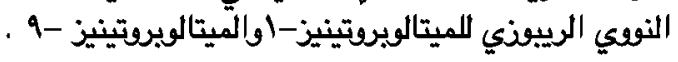

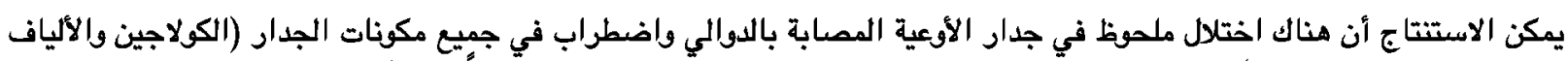

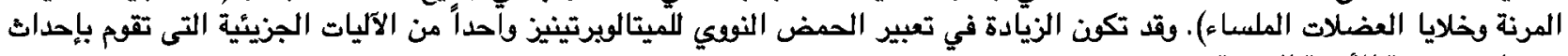
تغييرات في بنية الأوعية الدموية. 